\title{
What is the role of sentinel lymph node biopsy in the management of oral cancer in 2010 ?
}

\author{
Francisco J. Civantos · Sandro J. Stoeckli • Robert P. Takes · Julia A. Woolgar • \\ Remco de Bree • Vinidh Paleri $\cdot$ Kenneth O. Devaney $\cdot$ Alessandra Rinaldo • \\ Carl E. Silver · Vanni Mondin · Jochen A. Werner • Alfio Ferlito
}

Received: 1 February 2010 / Accepted: 4 February 2010 / Published online: 5 March 2010

(C) The Author(s) 2010. This article is published with open access at Springerlink.com

\section{Introduction}

Sentinel lymph node biopsy (SLNB) is a minimally invasive technique, performed in conjunction with radiotracer injection and lymphoscintigraphy. This allows the surgeon to identify and excise targeted upper echelon lymph nodes that drain the site of a primary malignancy for the laboratory detection of what would otherwise be subclinical nodal metastases. This technique offers a less invasive means of staging lymphatic basins in a patient with a primary malignancy, and permits detailed histological, immunohistochemical, and molecular examination of at least the first echelon (frequently second and rarely third) lymph node

This paper was written by members and invitees of the International Head and Neck Scientific Group (http://www.IHNSG.com).

\section{F. J. Civantos}

Department of Otolaryngology-Head and Neck Surgery,

Sylvester Comprehensive Cancer Center,

University of Miami, Miami, FL, USA

\section{S. J. Stoeckli}

Department of Otorhinolaryngology-Head and Neck Surgery,

Kantonsspital St. Gallen, St. Gallen, Switzerland

\section{R. P. Takes}

Department of Otolaryngology-Head and Neck Surgery,

Radboud University Nijmegen Medical Center,

Nijmegen, The Netherlands

\section{J. A. Woolgar}

Department of Oral Pathology,

School of Dental Sciences and Dental Hospital,

University of Liverpool, Liverpool, UK

R. de Bree

Department of Otolaryngology-Head and Neck Surgery,

VU University Medical Center, Amsterdam, The Netherlands basin for clinically occult micro- and conventional metastases (clinical stage N0). Use of this technique may potentially avoid overtreatment of patients with oral or oropharyngeal squamous cell carcinoma who undergo elective or opportune neck dissection and are subsequently declared pathologically N0, and hence reduce neck dissection-associated morbidity [1].

The sentinel node concept was first described by Cabanas [2] in 1977 for squamous cell carcinoma of the penis. In 1992, Morton et al. [3] reintroduced the concept of surgical sentinel lymph node sampling. Their landmark publication described their early prospective clinical experience with SLNB using blue dye in patients with clinically

V. Paleri

Department of Otolaryngology-Head and Neck Surgery, Newcastle upon Tyne Hospitals, Newcastle, UK

\section{K. O. Devaney}

Department of Pathology,

Allegiance Health, Jackson, MI, USA

A. Rinaldo $\cdot$ V. Mondin $\cdot$ A. Ferlito $(\square)$

Department of Surgical Sciences, ENT Clinic,

Azienda Ospedaliero-Universitaria di Udine,

Piazzale S. Maria della Misericordia, 33100 Udine, Italy

e-mail: a.ferlito@uniud.it

\section{E. Silver}

Departments of Surgery and Otolaryngology-Head and Neck Surgery, Albert Einstein College of Medicine, Montefiore Medical Center, Bronx, NY, USA

\section{J. A. Werner}

Department of Otolaryngology-Head and Neck Surgery, Philipp University, Marburg, Germany 
node-negative cutaneous malignant melanoma. The crucial introduction of radionuclides as the injected tracer can be attributed to Alex and Krag [4], who devised the current method involving nuclear imaging and a handheld gamma probe to identify sentinel lymph nodes, the technique that has become the standard of care for cutaneous malignant melanoma.

Oral squamous cell carcinoma (OSCC), like melanoma, is characterized by an anatomically stepwise progression of regional lymphatic metastases, and there has been an interest in the application of the technique to this disease. Formal lymphadenectomy of the draining lymphatic basins is a traditional option in OSCC. However, the current standard approach-selective neck dissection for deeply invasive lesions and "watchful waiting only" for superficial lesions-remains controversial. As increasing numbers of centers consider applying SLNB to OSCC patients, multiple other issues and questions arise regarding appropriate training and experience, patient selection, surgical and pathological techniques, and patient follow-up.

\section{Controversies in the management of the NO lymphatic basin for OSCC}

Patients with OSCC are usually examined by computed tomography (CT), magnetic resonance imaging (MRI), ultrasound-guided fine needle aspiration biopsy (USFNAB) or more recently positron emission tomography (PET) for evaluation of neck status, all of which have significant false-negative and false-positive rates. For detection of suspicious nodes appreciated on physical examination, USFNAB has proven to be the most accurate of these techniques [5], although the method is labor-intensive and operator dependent [6]. Nevertheless, these imaging techniques are not invariably capable of detecting nodal metastases.

Observation after removal of the primary tumor, "watchful waiting", with neck dissection only if clinical cervical metastases develop, has been proposed for patients with primary lesions considered at low risk for lymphatic metastases, based on small size (less than $2 \mathrm{~cm}$ ), minimal depth of invasion (for example less than $4 \mathrm{~mm}$ in primary tumors of the oral tongue), and favorable histological differentiation $[7,8]$. In these patients, close watching of the neck during follow-up has been recommended [9]. Depth of invasion has become the most widely accepted parameter for selecting patients for safe observation. However, a recent evaluation of a large population of patients with oral cancer who underwent SLNB found that tumor thickness was not a statistically significant predictor of positive sentinel nodes, whereas tumor differentiation, lymphovascular invasion, and invasive growth patterns were predictive [10]. This study was more robust than previous studies on this subject, as the true status of the neck was more accurately determined by step-serial sectioning and immunohistochemistry of the sentinel node. This study calls into question the widespread use of depth of invasion over all other variables as the primary means of allocating patients to "watchful waiting" versus neck dissection. In general, the presence of favorable histopathological characteristics in the primary tumor may indicate reduced risk, but does not negate the risk of metastases. Hence, no reliable tools are currently available that consistently achieve a high predictive value for occult metastasis.

Although there are no universally accepted guidelines, the predominant opinion is that a patient with a clinically N0 neck should have a neck dissection, if the risk of occult metastasis is more than approximately $15-20 \%$ [11-15]. This approach is supported primarily by retrospective studies that have demonstrated that a concomitant neck dissection is associated with a decreased rate of regional recurrence and distant metastases [15, 16]. Furthermore, one randomized study showed that patients with OSCC and a clinically NO neck, who did not have concomitant neck dissections, often presented later with neck metastases and incurable disease. Salvage surgery was successful in only $24 \%$ of these patients. However, patients with a prophylactic neck dissection had a reduction of recurrence in the neck alone, from 33 to $12 \%$ [17]. It should be kept in mind that the stage at which recurrences are detected is also dependent on the means and intensity of follow-up and may well determine the salvage rates. In addition, pathologic studies have shown that even small lymph node metastases, which are neither palpable nor obvious on imaging studies, can have extracapsular extension, including lymphovascular and perineural invasion, and other poor prognostic indicators [18-21]. Visualization and palpation of lymph nodes intraoperatively are unreliable predictors of nodal metastases by themselves [21]. In centers experienced in this procedure, USFNAB for follow-up enables early detection of a significant percentage of neck failures and a high salvage rate with therapeutic neck dissection [22]. However, this strategy may result in more extensive salvage surgeries for gross cervical metastases and more frequent postoperative radiotherapy, as compared to an approach that addresses the cervical lymphatics in conjunction with the initial surgical treatment.

The evaluation and treatment of lymphatic metastasis is further confounded by evidence that traditional surgical procedures, while accurate for the majority of patients, may miss involved lymph nodes in patients with variations in lymphatic anatomy or due to limitations in our ability to identify isolated tumor cells and micrometastases using traditional histopathologic techniques, where identifying minimal disease can be like finding the proverbial "needle in a haystack" [23]. 
In summary, for OSCC, the probability of microscopic involvement of lymph nodes than can be estimated based on clinical findings and routine histopathology is at best an approximation. For oral cavity malignancies, the reported $20-40 \%$ risk of occult metastases must be weighed against the morbidity of dissecting the majority of necks that are not truly involved. The need for a better diagnostic technique to identify subclinical cervical metastases and guide the treatment of these patients ultimately has lead to the development of SLNB. Another development reducing the probability of occult metastasis and facilitating more adequate treatment decisions may be the availability of biological tests performed on the primary tumor specimen, but the promising early results of such profiles need to be validated [24]. The decision to observe a patient at moderate risk for lymphatic metastases who has a clinically negative neck, which was sensible in the era of more radical neck procedures, is made more difficult with the current practice favoring selective, functional neck dissections, and might become even less common if the evidence base for SLNB becomes stronger.

\section{SLNB for OSCC}

Between 1996 and 2000, multiple centers initiated single institutional trials studying SLNB for OSCC [25-29]. More than 60 single institution trials, two international conference consensus documents, a meta-analysis, and recent joint practice guidelines have since been published concerning this topic [30-32]. A large multi-institutional pathologic validation trial has supported previous pathologic validation trials and established that the sentinel node concept applies to OSCC as it does to cutaneous melanoma and breast cancer. The pathologic status of the sentinel node correlated well with the status of subsequent immediate formal lymphadenectomy [33]. Several large prospective clinical observational trials on SLNB in OSCC, with selective neck dissection reserved only for proven positive lymphatic metastases, have been conducted to date [34, 35]. Three prospective studies of SLNB as primary neck management for stage I and II oral cavity cancer [Sentinel Node European Trial (SENT), the Danish National Group Trial (DAHANCA 22), and the Brazilian Head and Neck Group] are in progress.

The consensus in the literature regarding this topic is that: (1) the predictive value of a negative sentinel lymph node varies between 90 and 100\%; (2) step-serial sectioning and immunohistochemistry is essential in proper evaluation of the sentinel lymph node; (3) this use of step-serial sectioning and immunohistochemistry can significantly improve the negative predictive value of this technique; (4) significant upstaging of the lymphatic basins occurs with this technique relative to standard formal lymphadenectomy; (5) unexpected patterns of lymphatic drainage can indeed occur, including unanticipated contralateral drainage to nodes that might be missed with standard lymphadenectomies, and (6) if immediate assessment of lymph nodes using frozen section, imprint cytology or molecular biological techniques reliably show a sentinel lymph node metastasis, a neck dissection can be performed at the same sitting. Nevertheless, significant concerns exist regarding the need for staged surgery in the small percentage of patients whose sentinel nodes are found to be positive at a later date (after formal processing including immunohistochemistry, which typically adds 1-2 days to the preparation of the final pathology report), as well as with regard to the risk of false-negatives in what would otherwise be a highly curable condition. The optimum management of the neck that harbors only a single micrometastasis or even isolated tumor cells is, as yet, uncertain. Some have conjectured that removal of just the sentinel node may be sufficient in these patients, though this has not been formally studied.

\section{Morbidity of SLNB versus selective neck dissection}

Selective neck dissection was developed primarily as a means of reducing the morbidity related to more radical approaches to the neck. However, significant morbidity remains even with the selective procedure, including adhesive capsulitis of the shoulder (which can occur even after only transient weakness of the trapezius muscle), lip movement asymmetry, and contour changes in the neck. This has been documented in multiple quality of life studies and functional assessments [36-39]. In theory, an inexperienced operator could create more injury through a narrow exposure approach to the neck than a formal dissection. However, several studies have documented negligible morbidity with SLNB in the hands of experienced head and neck surgeons [40]. No contour changes occur, and capsular dissection of lymph nodes minimizes the need for nerve dissection. For patients with minimal disease detected after final pathological analysis, the option of early re-exploration allows for completion neck dissection without increased morbidity. In a recent study, Murer et al. [41] compared complication rate, morbidity, and postoperative shoulder function in a large cohort undergoing either SLNB or elective neck dissection. Subjective impairment was assessed with the neck dissection impairment index (NDII) questionnaire. The functional status was evaluated by the assessment of shoulder function with the modified individual relative Constant score. Perhaps not surprisingly, SLNB was associated with significantly fewer complications, less subjective neck and shoulder impairment, and better objective shoulder function than elective neck dissection. SLNB 
has recently been shown to be associated with better quality of life compared to selective neck dissection [42].

Apart from the eventual functional and oncological outcomes, the morbidity and costs involved in the several strategies for treatment of the N0 neck should be factored in before arriving at more definitive answers.

\section{Discussion}

The sentinel lymph node procedure has become a well investigated and elaborated technique and much has already been accomplished. However, some issues still need further development. An important issue to be resolved before SLNB can be advocated more strongly for the majority of deeply invasive oral cancers is our limited ability to achieve immediate diagnosis of positive sentinel nodes. The use of frozen section is controversial as tissue that has been frozen, then thawed, and then processed for routine light microscopic examination may be compromised by this handling, and might not represent the true status of the entire specimen. Although some groups do advocate frozen section for immediate conversion to selective neck dissection, with reduction in the need for reoperation [35, 43, 44], this is still a matter of debate.

To be clear, there are several inherent potential pitfalls to be aware of, when selecting a definitive method of triaging patients for further treatment: first, there is evidence that skip metastases-i.e., metastases that skip over the first echelon draining node to lodge in nodes further downstream-do exist, which means that sampling sentinel nodes alone can yield false-negative results in those infrequent patients with skip metastases [45]. Therefore, guidelines to define which second echelon "sentinel" nodes have to be harvested are needed. Such guidelines are not incorporated in the joint practice guidelines [1], and multiple algorithms exist. Second, routine light microscopy, whether at the time of surgery (frozen section examination) or after fixation, runs a risk of overlooking tiny metastatic deposits. Light microscopic examination is, in essence, a sampling process, which means that tiny deposits made up of a limited number of tumor cells may be left behind in the tissue not sampled (or even in the portion of the tissue block not actually cut) [46]. This may be improved by step-serial sectioning but cannot entirely be circumvented. Finally, soft tissue tumor deposits (i.e., tumor deposits which are not clearly associated with an encompassing lymph node) may be encountered in neck dissection specimens, and would not be identified by sentinel node sampling procedures [47].

For the patient with a microscopic metastatic deposit in the sentinel node, not detected at the initial SLNB, we are dealing with issues that potentially include re-exploration and dissection of functionally important nerves in a recently operated wound. If the final pathology report can be made available to permit the patient to be taken back to surgery within 7 days, the concern is lessened, but a timely pathology report can be logistically challenging in some centers. Ultimately, rapid reverse transcriptase-polymerase chain reaction assessment of nodes may provide immediate information regarding the status of the sentinel node, but this technique has not yet been validated for clinical use [48].

As selective neck dissection has traditionally been advocated if the risk of subclinical metastases exceeds 15-20\% [11-15], we are applying "watchful waiting" to a group of patients who still have significant risk, including many lip lesions, thin $\mathrm{T} 1$ cancers in other areas of the oral cavity, and other lesions with low probability of metastasis. For an increasing number of head and neck surgeons, SLNB is considered a reasonable option for patients with low but not negligible risk of harboring lymphatic metastases, and selective neck dissection remains the mainstay for patients with higher risk of subclinical nodal involvement. However, as discussed earlier, the appropriate population for SLNB, with an intermediate risk of occult metastasis, needs to be defined more clearly, and tools for risk assessment are currently relatively limited. An alternative approach is to offer SLNB to all patients with OSCC. However, if the neck has to be entered for primary tumor resection or free flap reconstruction in larger tumors, it may be more rational to perform a selective or opportune neck dissection in these cases.

Multiple authors have emphasized the significant learning curve that exists for SLNB. In the American College of Surgeons Oncology Group pathologic validation trial, there was a statistically significant difference in the negative predictive value of SLNB between experienced surgeons and beginning surgeons, even though the latter had performed five cases and participated in an animal laboratory [33]. It is important for surgeons to receive initial instruction in the proper handling of the gamma probe. The surgeon should subsequently gain experience in the use of the gamma probe by performing SLNB prior to a planned completion neck dissection. This can be performed in a "gamma probeguided neck dissection", in which the sentinel node is examined more meticulously using step-serial sectioning and immunohistochemistry to obtain a more reliable histopathological examination. This procedure offers benefits to the patient, and is more than just a means of gaining experience. Significant upstaging of the cervical nodes can be anticipated by virtue of the gamma probe-assisted procedure $[23,39]$. Clearly these patients can be more intelligently followed and treated when we understand the true status of the neck. Thus, SLNB in the context of a completion selective neck dissection offers the opportunity for more accurate staging of minimal, but significant, neck disease, and can be offered to patients in good conscience. 
Equally important is the occasional unpredictability of lymphatic pathways, which is well recognized for cutaneous lesions, and also occurs with oral lesions. In fact for lesions not involving the midline but within a few centimeters of it, gamma probe-guided surgery may provide a superior solution for these patients in whom we often struggle with decisions regarding contralateral neck management.

The SLNB remains an exciting area of development for OSCC and other head and neck mucosal lesions. Technologies are rapidly improving, and surgeons are becoming more experienced. It is very likely that the use of SLNB will increase as technological refinements [e.g. single photon emission $\mathrm{CT}$ and CT (SPECT/CT), intraoperative gamma camera and fluorescence imaging] occur, and that it will be applied in an increasingly wider group of mucosal lesions.

\section{Conclusions}

The technique of SLNB offers the potential for more anatomically accurate surgery based on each patient's unique lymphatic drainage pattern. It has been demonstrated to have an excellent safety profile with good sensitivity in identifying occult neck metastases. Use of this technique can potentially allow selection of patients for selective neck dissection, sparing them the morbidity of overtreatment and offer better quality of life. Selective neck dissection has only a $5 \%$ chance of not securing control of neck metastases, and it may well transpire on longitudinal studies that SLNB has similar oncologic efficacy. However, in the absence of reproducible level-1 evidence and the existence of a defined learning curve, centers commencing SLNB should offer this procedure within a trial setting, and the results should be closely monitored. In centers with adequate experience, SLNB can be offered to appropriate patients with nodal negative T1 and T2 OSCC for accurate histopathologic staging of the neck, with periodic audit of oncologic outcomes.

Open Access This article is distributed under the terms of the Creative Commons Attribution Noncommercial License which permits any noncommercial use, distribution, and reproduction in any medium, provided the original author(s) and source are credited.

\section{References}

1. Alkureishi LW, Burak Z, Alvarez JA et al (2009) Joint practice guidelines for radionuclide lymphoscintigraphy for sentinel node localization in oral/oropharyngeal squamous cell carcinoma. Ann Surg Oncol 16:3190-3210

2. Cabanas RM (1977) An approach for the treatment of penile carcinoma. Cancer 39:456-466
3. Morton DL, Wen D-R, Wong JH et al (1992) Technical details of intraoperative lymphatic mapping for early stage melanoma. Arch Surg 127:392-399

4. Alex JC, Krag DN (1996) The gamma-probe-guided resection of radiolabeled primary lymph nodes. Surg Oncol Clin N Am 5:3341

5. de Bondt RB, Nelemans PJ, Hofman PA et al (2007) Detection of lymph node metastases in head and neck cancer: a meta-analysis comparing US, USgFNAC, CT and MR imaging. Eur J Radiol 64:266-272

6. Takes RP, Knegt PPM, Manni JJ et al (1996) Regional metastasis in head and neck squamous cell cancer: the value of ultrasound with UGFNAB revised. Radiology 198:819-823

7. Shear M, Hawkins DM, Farr HW (1976) The prediction of lymph node metastases from oral squamous carcinoma. Cancer 37:19011907

8. Martínez-Gimeno C, Rodríguez EM, Vila CN, Varela CL (1995) Squamous cell carcinoma of the oral cavity: a clinicopathologic scoring system for evaluating risk of cervical lymph node metastasis. Laryngoscope 105:728-733

9. Pillsbury HC, Clark M (1997) A rationale for therapy of the N0 neck. Laryngoscope 107:1294-1315

10. Goerkem M, Braun J, Stoeckli SJ (2010) Evaluation of clinical and histomorphological parameters as potential predictors of occult metastases in sentinel lymph nodes of early squamous cell carcinoma of the oral cavity. Ann Surg Oncol 17:527-535

11. Pitman KT, Johnson JT, Myers EN (1997) Effectiveness of the selective neck dissection for the management of the clinically negative neck. Arch Otolaryngol Head Neck Surg 123:917-922

12. McGuirt WF Jr, Johnson JT, Myers EN, Rothfield R, Wagner R (1995) Floor of mouth carcinoma. The management of the clinically negative neck. Arch Otolaryngol Head Neck Surg 121:278282

13. Shingaki S, Kobayashi T, Suzuki I, Kohno M, Nakajima T (1995) Surgical treatment of stage I and II oral squamous cell carcinomas: analysis of causes of failure. Br J Oral Maxillofac Surg 33:304308

14. Shah JP, Andersen PE (1995) Evolving role of modifications in neck dissection for oral squamous carcinoma. Br J Oral Maxillofac Surg 33:3-8

15. Clayman GL, Frank DK (1998) Selective neck dissection of anatomically appropriate levels is as efficacious as modified radical neck dissection for elective treatment of the clinically negative neck in patients with squamous cell carcinoma of the upper respiratory and digestive tracts. Arch Otolaryngol Head Neck Surg 124:348-352

16. Yuen APW, Wei WI, Wong YM, Tang KC (1997) Elective neck dissection versus observation in the treatment of early oral tongue carcinoma. Head Neck 19:583-588

17. Kligerman J, Lima RA, Soares JR et al (1994) Supraomohyoid neck dissection in the treatment of $\mathrm{T} 1 / \mathrm{T} 2$ squamous cell carcinoma of oral cavity. Am J Surg 168:391-394

18. Braams JW, Pruim J, Freling NJM et al (1995) Detection of lymph node metastases of squamous-cell cancer of the head and neck with FDG-PET and MRI. J Nucl Med 36:211-216

19. Alvi A, Johnson JT (1996) Extracapsular spread in the clinically negative neck (N0): implications outcome. Otolaryngol Head Neck Surg 114:65-70

20. Mamelle G, Pampurik J, Luboinski B, Lancar R, Lusinchi A, Bosq J (1994) Lymph node prognostic factors in head and neck squamous cell carcinomas. Am J Surg 168:494-498

21. Rassekh CH, Johnson JT, Myers EN (1995) Accuracy of intraoperative staging of the N0 neck in squamous cell carcinoma. Laryngoscope 105:1334-1336

22. Nieuwenhuis EJ, Castelijns JA, Pijpers R et al (2002) Wait-andsee policy for the N0 neck in early-stage oral and oropharyngeal 
squamous cell carcinoma using ultrasonography-guided cytology: is there a role for identification of the sentinel node? Head Neck 24:282-289

23. Ross GL, Shoaib T, Soutar DS et al (2002) The use of sentinel node biopsy to upstage the clinically N0 neck in head and neck cancer. Arch Otolaryngol Head Neck Surg 128:1287-1290

24. Roepman P, Wessels LF, Kettelarij N et al (2005) An expression profile for diagnosis of lymph node metastases from primary head and neck squamous cell carcinomas. Nat Genet 37:182-186

25. Shoaib T, Soutar DS, MacDonald DG et al (2001) The accuracy of head and neck carcinoma sentinel lymph node biopsy in the clinically N0 neck. Cancer 91:2077-2083

26. Civantos FJ, Gomez C, Duque C et al (2003) Sentinel node biopsy in oral cavity cancer: correlation with PET scan and immunohistochemistry. Head Neck 25:1-9

27. Stoeckli SJ, Steinert H, Pfaltz M, Schmid S (2001) Sentinel lymph node evaluation in squamous cell carcinoma of the head and neck. Otolaryngol Head Neck Surg 125:221-226

28. Zitsch RP III, Todd DW, Renner GJ, Singh A (2000) Intraoperative radiolymphoscintigraphy for detection of occult nodal metastasis in patients with head and neck squamous cell carcinoma. Otolaryngol Head Neck Surg 122:662-666

29. Taylor RJ, Wahl RL, Sharma PK et al (2001) Sentinel node localization in oral cavity and oropharynx squamous cell cancer. Arch Otolaryngol Head Neck Surg 127:970-974

30. Stoeckli SJ, Alkureishi LW, Ross GL (2009) Sentinel node biopsy for early oral and oropharyngeal squamous cell carcinoma. Eur Arch Otorhinolaryngol 266:787-793

31. Stoeckli SJ, Pfaltz M, Ross GL et al (2005) The second international conference on sentinel node biopsy in mucosal head and neck cancer. Ann Surg Oncol 12:919-924

32. Paleri V, Rees G, Arullendran P, Shoaib T, Krishman S (2005) Sentinel node biopsy in squamous cell cancer of the oral cavity and oral pharynx: a diagnostic meta-analysis. Head Neck 27:739-747

33. Civantos FJ, Zitsch RP, Schuller DE et al (2010) Sentinel lymph node biopsy accurately stages the regional lymph nodes for T1-T2 oral squamous cell carcinomas: results of a prospective multiinstitutional trial. J Clin Oncol [Epub ahead of print]

34. Ross GL, Soutar DS, MacDonald DG et al (2004) Sentinel node biopsy in head and neck cancer: preliminary results of a multicenter trial. Ann Surg Oncol 11:690-696

35. Stoeckli SJ (2007) Sentinel node biopsy for oral and oropharyngeal squamous cell carcinoma of the head and neck. Laryngoscope 117:1539-1551
36. Chepeha DB, Taylor RJ, Chepeha JC et al (2002) Functional assessment using Constant's Shoulder Scale after modified radical and selective neck dissection. Head Neck 24:432-436

37. Kuntz AL, Weymuller EA Jr (1999) Impact of neck dissection on quality of life. Laryngoscope 109:1334-1338

38. Rogers SN, Ferlito A, Pellitteri PK, Shaha AR, Rinaldo A (2004) Quality of life following neck dissections. Acta Otolaryngol 124:231-236

39. van Wilgen CP, Dijkstra PU, Nauta JM, Vermey A, Roodenburg JL (2003) Shoulder pain and disability in daily life, following supraomohyoid neck dissection: a pilot study. J Craniomaxillofac Surg 31:183-186

40. Civantos FJ, Moffat FL, Goodwin WJ (2006) Lymphatic mapping and sentinel lymphadenectomy for 106 head and neck lesions: contrasts between oral cavity and cutaneous malignancy. Laryngoscope 112(Suppl 109):1-15

41. Murer K, Huber G, Haile SR, Stoeckli SJ (2010) Comparison of morbidity between sentinel node biopsy and elective neck dissection for treatment of the $\mathrm{N} 0$ neck in patients with oral squamous cell carcinoma. Head Neck (in press)

42. Schiefke F, Akdemir M, Weber A et al (2009) Function, postoperative morbidity, and quality of life after cervical sentinel node biopsy and after selective neck dissection. Head Neck 31:503-512

43. Terada A, Hasegawa $Y$, Yatabe $Y$ et al (2008) Intraoperative diagnosis of cancer metastasis in sentinel lymph node of oral cancer patients. Oral Oncol 44:838-843

44. Tschopp L, Nuyens M, Stauffer E, Krause T, Zbären P (2005) The value of frozen section analysis of the sentinel lymph node in clinically N0 squamous cell carcinoma of the oral cavity and oropharynx. Otolaryngol Head Neck Surg 132:99-102

45. Ferlito A, Shaha AR, Rinaldo A, Pelliteri PK, Mondin V, Byers RM (2002) "Skip metastases" from head and neck cancers. Acta Otolaryngol 122:788-791

46. Ferlito A, Rinaldo A (2000) Controversies in the treatment of N0 neck in laryngeal cancer: neck dissection, no surgery or sentinel lymph node biopsy? ORL J Otorhinolaryngol Relat Spec 62:287289

47. Jose J, Ferlito A, Rodrigo JP, Devaney KO, Rinaldo A, MacLennan K (2007) Soft tissue deposits from head and neck cancer: an under-recognised prognostic factor? J Laryngol Otol 121:11151117

48. Ferris RL, Xi L, Raja S et al (2005) Molecular staging of cervical lymph nodes in squamous cell carcinoma of the head and neck. Cancer Res 65:2147-2156 